\title{
ISOMORPHISMS OF TRIFORM SEMIGROUPS
}

\author{
KENNETH D. MAGILL, Jr. \\ (Received 14 March 1968; revised 20 May 1968)
}

\section{Introduction}

For any point $x$, we use the symbol $\langle x\rangle$ to denote $\{(x, x)\}$. By a triform, we mean a triple $\left(X, \mathscr{F}_{X}, \mathscr{S}[X]\right)$ where $X$ is a nonempty set, $\mathscr{F}_{X}$ is a family of subsets of $X, \mathscr{S}[X]$ is a family of subsets of $X \times X$ and the following conditions are satisfied:

(1.1) $\langle x\rangle \in \mathscr{S}[X]$ for each $x \in X$.

(1.2) $H \in \mathscr{F}_{X}$ if and only if $H \times\{x\} \in \mathscr{S}[X]$ for some $x \in X$.

(1.3) $A \circ B \in \mathscr{P}[X]$ for each $A, B \in \mathscr{P}[X]$ where $A \circ B$ is defined by $A \circ B=\{(x, y) \in X \times X:(x, z) \in B$ and $(z, y) \in A$ for some $z \in X\}$.

It follows immediately from conditions (1.1) and (1.2) that all singletons belong to $\mathscr{F}_{X}$. Condition (1.3) guarantees that, under composition, $\mathscr{S}[X]$ is a semigroup of binary relations on the set $X$. We will refer to $\mathscr{S}[X]$ as the semigroup of the triform $\left(X, \mathscr{F}_{X}, \mathscr{S}[X]\right)$ or, more often, simply as a triform semigroup.

The main result of this paper is Theorem (2.2) of Section 2 which describes all isomorphisms between triform semigroups. This result is applied in Section 3 to semigroups of closed relations on certain topological spaces. Among other things, it follows that two such semigroups are isomorphic if and only if the corresponding spaces are homeomorphic. Corollaries (3.4) and (3.5) generalize the two results in [4].

\section{The isomorphism theorem}

Before stating the isomorphism theorem, it will be convenient to have the following

Definition (2.1). Let $\left(X, \mathscr{F}_{X}, \mathscr{S}[X]\right)$ and $\left(Y, \mathscr{F}_{Y}, \mathscr{S}[Y]\right)$ be two triforms. A bijection $h$ from $X$ onto $Y$ is referred to as a trimorphism if $h[A] \in \mathscr{F}_{\boldsymbol{Y}}$ for each $A \in \mathscr{F}_{\boldsymbol{X}}$ and $h^{-1}[A] \in \mathscr{F}_{\boldsymbol{X}}$ for each $A \in \mathscr{F}_{\boldsymbol{Y}}$.

THEOREM (2.2). The following statements concerning a bijection $\varphi$ from a triform semigroup $\mathscr{S}[X]$ onto a triform semigroup $\mathscr{S}[Y]$ are equivalent: 
(2.2.1) $\varphi$ is an isomorphism.

(2.2.2) There exists a trimorphism $h$ from $X$ onto $Y$ such that

$$
\varphi(A)=h \circ A \circ h^{-1}
$$

for each $A \in \mathscr{S}[X]$.

(2.2.3) There exists a trimorphism $h$ from $X$ onto $Y$ such that

$$
\varphi(A)=\{(h(x), h(y)):(x, y) \in A\}
$$

for each $A \in \mathscr{S}[X]$.

It seems to be advantageous to prove a number of preliminary lemmas about the triform $\left(X, \mathscr{F}_{X}, \mathscr{S}[X]\right)$ before proving the theorem. First of all, let us observe that if $X$ has two distinct points, $x$ and $y$, then both $\langle x\rangle$ and $\langle y\rangle$ belong to $\mathscr{P}[X]$. Consequently, condition (1.3) implies $\langle\boldsymbol{x}\rangle \circ\langle y\rangle=\phi$ belongs to $\mathscr{S}[X]$. When the empty set is to be regarded as an element of $\mathscr{S}[X]$, it will be denoted by the letter $E$. One easily verifies that if $E \in \mathscr{S}[X]$, then $E$ is the zero of $\mathscr{S}[X]$. We state these observations formally as

LEMMA (2.3). If $E \in \mathscr{S}[X]$, then $E$ is the zero of $\mathscr{S}[X]$. Furthermore, $E$ belongs to $\mathscr{P}[X]$ if $X$ has more than one point.

If $X$ consists of only one point $p, \mathscr{S}[X]$ need not contain $E$, it might possibly consist solely of the element $\langle p\rangle$. For this particular case, it is a straightforward matter to verify that the statement of Theorem (2.2) is valid. It is therefore sufficient to verify the statement for the case when both $X$ and $Y$ have more than one point and hence both $\mathscr{S}[X]$ and $\mathscr{S}[Y]$ contain $E$. Consequently, we assume in the lemmas leading up to the proof of Theorem (2.2) and in the proof itself that both $X$ and $Y$ have more than one point.

Definition (2.4). For any relation $A \in \mathscr{S}[X]$, we let

$$
\mathscr{D}(A)=\{x \in X:(x, y) \in A \text { for some } y \in X\}
$$

and

$$
\mathscr{R}(A)=\{x \in X:(y, x) \in A \text { for some } y \in X\} .
$$

The next lemma is easily proved.

Lemma (2.5). For $A, B \in \mathscr{S}[X], A \circ B=E$ if and only if

$$
\mathscr{D}(A) \cap \mathscr{R}(B)=\phi .
$$

Lemma (2.6). Suppose $A, B \in \mathscr{S}[X]$. Then $\mathscr{D}(A) \subset \mathscr{D}(B)$ if and only if for each $C \in \mathscr{S}[X], A \circ C \neq E$ implies $B \circ C \neq E$.

Proof. Suppose $\mathscr{D}(A) \subset \mathscr{D}(B)$ and $A \circ C \neq E$. Then there exist points $x, y, z \in X$ such that $(x, y) \in C$ and $(y, z) \in A$. Then $y \in \mathscr{D}(A) \subset \mathscr{D}(B)$ which implied that $(y, v) \in B$ for some $v \in X$. Consequently, $(x, v) \in B \circ C$, i.e., $B \circ C \neq E$. 
Now suppose $\mathscr{D}(A) \notin \mathscr{D}(B)$. Then there exists a point $p \in \mathscr{D}(A)-\mathscr{D}(B)$. Then $\langle p\rangle \in \mathscr{S}[X]$ and the desired contradiction is obtained by noting that

while

$$
A \circ\langle p\rangle \neq E
$$$$
B \circ\langle p\rangle=E \text {. }
$$

In a similar manner, one verifies

Lemma (2.7). $\mathscr{R}(A) \subset \mathscr{R}(B)$ if and only it for every $C \in \mathscr{S}[X], C \circ A \neq E$ implies $C \circ B \neq E$.

Lemma (2.8). $\mathscr{D}(A) \subset \mathscr{R}(B)$ if and only if for each $C \in \mathscr{P}[X]-\{E\}$, $\mathscr{D}(C) \subset \mathscr{D}(A)$ implies $C \circ B \neq E$.

Proof. First suppose $\mathscr{D}(A) \subset \mathscr{R}(B), C \in \mathscr{S}[X]-\{E\}$, and $\mathscr{D}(C) \subset \mathscr{D}(A)$. Then there exists a point $x \in X$ such that $x \in \mathscr{D}(C) \subset \mathscr{D}(A) \subset \mathscr{R}(B)$. This implies that $(x, y) \in C$ and $(z, x) \in B$ for some $y, z \in X$. Consequently, $(z, y) \in C \circ B$, i.e., $C \circ B \neq E$.

On the other hand, suppose $\mathscr{D}(A) \notin \mathscr{R}(B)$. Then there exists a point $x \in \mathscr{D}(A)-\mathscr{R}(B)$. Since $\langle x\rangle \in \mathscr{S}[X]-\{E\}$ and $\mathscr{D}(\langle x\rangle) \subset \mathscr{D}(A)$ but $\langle x\rangle \circ B=E$, we have reached a contradiction.

Similarly, one proves

LEMma (2.9). $\mathscr{R}(A) \subset \mathscr{D}(B)$ if and only if for each $C \in \mathscr{S}[X]-\{E\}$, $\mathscr{R}(C) \subset \mathscr{R}(A)$ implies $B \circ C \neq E$.

Lemma (2.10). $\mathscr{R}(A)$ consists of one point if and only if there exists precisely one $B \in \mathscr{S}[X]-\{E\}$ such that $\mathscr{D}(B) \subset \mathscr{R}(A)$ and $\mathscr{R}(B) \subset \mathscr{R}(A)$.

Proof. Suppose $\mathscr{R}(A)$ consists of one point $p$. Then $\langle p\rangle \in \mathscr{S}[X]-\{E\}$, $\mathscr{D}(\langle p\rangle) \subset \mathscr{R}(A), \mathscr{R}(\langle p\rangle) \subset \mathscr{R}(A)$ and $\langle p\rangle$ is the only element in $\mathscr{S}[X]$ which satisfies these conditions.

Suppose, however, that $\mathscr{R}(A)$ does not consist of one point. If we assume $\mathscr{R}(A)=\phi$, we get a contradiction immediately. The remaining case is where $\mathscr{R}(A)$ contains at least two distinct points $p$ and $q$. Then both $\langle p\rangle$ and $\langle q\rangle$ belong to $\mathscr{S}[X]-\{E\}$. Furthermore, $\mathscr{D}(\langle p\rangle) \subset \mathscr{R}(A)$, $\mathscr{D}(\langle q\rangle) \subset \mathscr{R}(A), \mathscr{R}(\langle p\rangle) \subset \mathscr{R}(A)$ and $\mathscr{R}(\langle q\rangle) \subset \mathscr{R}(A)$. This, however, is a contradiction since $\langle p\rangle$ and $\langle q\rangle$ are distinct.

In much the same way, one proves:

LeMma (2.11). $\mathscr{D}(A)$ consists of one point if and only if there exists precisely one $B \in \mathscr{S}[X]-\{E\}$ such that $\mathscr{D}(B) \subset \mathscr{D}(A)$ and $\mathscr{R}(B) \subset \mathscr{D}(A)$.

One easily verifies the following two lemmas.

Lemma (2.12). Let $A$ be an element of $\mathscr{S}[X]$. Then $A=\langle x\rangle$ for some $x \in X$ if and only if $\mathscr{D}(A)$ consists of one point, $\mathscr{R}(A)$ consists of one point and $A \circ A \neq E$. 
Lemma (2.13). $(x, y) \in A$ if and only if $\langle y\rangle \circ A \circ\langle x\rangle \neq E$.

Now we are finally in a position to prove the theorem. First of all, it is a routine matter to show that for any bijection $h$ from $X$ onto $Y$ and any $A \in \mathscr{S}[X]$,

$$
h \circ A \circ h^{-1}=\{(h(x), h(y)):(x, y) \in A\} .
$$

From this it follows that (2.2.2) and (2.2.3) are equivalent. Furthermore, it is easily seen that (2.2.2) implies (2.2.1). Consequently, our task is complete when we show that (2.2.1) implies (2.2.2). We first define the mapping $h$. Let any $x \in X$ be given. Now lemmas (2.3) to (2.12) together characterize $\langle x\rangle$ algebraically and it follows that $\varphi\langle x\rangle=\langle y\rangle$ for some unique $y \in Y$. Define the function $h$ by $h(x)=y$. The function $h$ is a bijection since $\varphi$ is an isomorphism. Furthermore, we note that

$$
\varphi\langle x\rangle=\langle h(x)\rangle
$$

for each $x \in X$.

Now let any $A \in \mathscr{S}[X]$ be given. We will show that

$$
\varphi(A)=h \circ A \circ h^{-1} \text {. }
$$

Suppose $(x, y) \in \varphi(A)$. Then by Lemma (2.13),

But

$$
\langle y\rangle \circ \varphi(A) \circ\langle\boldsymbol{x}\rangle \neq E \text {. }
$$

$$
\begin{aligned}
& \langle y\rangle \circ \varphi(A) \circ\langle x\rangle \\
& \quad=\varphi\left\langle h^{-1}(y)\right\rangle \circ \varphi(A) \circ \varphi\left\langle h^{-1}(x)\right\rangle=\varphi\left(\left\langle h^{-1}(y)\right\rangle \circ A \circ\left\langle h^{-1}(x)\right\rangle\right)
\end{aligned}
$$

which implies that

$$
\left\langle h^{-1}(y)\right\rangle \circ A \circ\left\langle h^{-1}(x)\right\rangle \neq E .
$$

Using Lemma (2.13) again, we conclude that

$$
\left(h^{-1}(x), h^{-1}(y)\right) \in A .
$$

This, together with the fact that $\left(x, h^{-1}(x)\right) \in h^{-1}$ and $\left(h^{-1}(y), y\right) \in h$ implies $(x, y) \in h \circ A \circ h^{-1}$.

Now suppose $(x, y) \in h \circ A \circ h^{-1}$. Then there exist points $v$ and $w$ in $X$ such that $(x, v) \in h^{-1},(v, w) \in A$ and $(w, y) \in h$. Since $h(v)=x$ and $h(w)=y$, it follows that

$$
\varphi\langle v\rangle=\langle x\rangle \text { and } \varphi\langle w\rangle=\langle y\rangle .
$$

Furthermore, since $(v, w) \in A$, it follows that

$$
\langle w\rangle \circ A \circ\langle v\rangle \neq E \text {. }
$$


But then

$$
\begin{aligned}
E & \neq \varphi(\langle w\rangle \circ A \circ\langle v\rangle) \\
& =\varphi\langle w\rangle \circ \varphi(A) \circ \varphi\langle v\rangle \\
& =\langle y\rangle \circ \varphi(A) \circ\langle x\rangle
\end{aligned}
$$

which, by Lemma (2.13) implies $(x, y) \in \varphi(A)$. This completes the verification of (2.13.2).

Now let any $H \in \mathscr{F}_{X}$ be given. According to (1.2), $H \times\{x\} \in \mathscr{P}[X]$ for some $x \in X$. Thus, (2.13.2) implies that

But

$$
h \circ(H \times\{x\}) \circ h^{-1} \in \mathscr{S}[Y] .
$$

$$
h \circ(H \times\{x\}) \circ h^{-1}=h[H] \times\{h(x)\}
$$

and it follows from (1.2) that $h[H] \in \mathscr{F}_{Y}$. In a similar manner, one shows that $h^{-1}[H] \in \mathscr{F}_{X}$ for each $H \in \mathscr{F}_{Y}$. This implies that $h$ is a trimorphism and the proof is complete.

COROLLARY (2.14). The automorphism group of a triform semigroup $\mathscr{S}[X]$ is isomorphic to a subgroup of the group, under composition, of all trimorphisms mapping $X$ onto $X$.

Proof. Let $\mathscr{A}$ denote the automorphism group of $\mathscr{S}[X]$ and let $\mathscr{G}$ denote the group of all trimorphisms mapping $X$ onto $X$. We define a mapping $\Phi$ from $\mathscr{A}$ into $\mathscr{G}$. Let $\varphi \in \mathscr{A}$ be given. According to Theorem (2.2), there exists a (necessarily unique) $h \in \mathscr{G}$ such that

$$
\varphi(A)=h \circ A \circ h^{-1}
$$

for each $A \in \mathscr{S}[X]$. We define

$$
\Phi(\varphi)=h .
$$

Let $\Phi\left(\varphi_{1}\right)=h_{1}$ and $\Phi\left(\varphi_{2}\right)=h_{2}$ for two automorphisms $\varphi_{1}, \varphi_{2}$ of $\mathscr{S}[X]$. Then for any $A \in \mathscr{S}[X]$,

$$
\begin{aligned}
\left(\varphi_{1} \circ \varphi_{2}\right)(A) & =\varphi_{1}\left(\varphi_{2}(A)\right) \\
& =\varphi_{1}\left(h_{2} \circ A \circ h_{2}^{-1}\right)=h_{1} \circ h_{2} \circ A \circ h_{2}^{-1} \circ h_{1}^{-1} \\
& =\left(h_{1} \circ h_{2}\right) \circ A \circ\left(h_{1} \circ h_{2}\right)^{-1} .
\end{aligned}
$$

It follows that

$$
\Phi\left(\varphi_{1} \circ \varphi_{2}\right)=h_{1} \circ h_{2}=\Phi\left(\varphi_{1}\right) \circ \Phi\left(\varphi_{2}\right)
$$

i.e., $\Phi$ is a homomorphism.

In order to see that $\Phi$ is injective, suppose $\Phi(\varphi)=i$, the identity map on $X$. Then

$$
\varphi(A)=i \circ A \circ i^{-1}=A
$$


for each $A \in \mathscr{S}[X]$, i.e., $\varphi$ is the identity automorphism. Thus the kernel of $\Phi$ is the identity and the proof is complete.

The following example shows that the automorphism group $\mathscr{A}$ of $\mathscr{S}[X]$ need not actually be isomorphic to the group $\mathscr{G}$ of all trimorphisms on $X$.

Example (2.15). Let $X$ denote the unit interval, let $\mathscr{F}_{X}$ denote the power set of $X$ and let $\mathscr{S}[X]$ denote the family of all continuous functions whose domain is a subset of $X$ and whose range is a subset of $X$. We assume the empty function also belongs to $\mathscr{S}[X]$.

One easily verifies that $\left(X, \mathscr{F}_{X}, \mathscr{S}[X]\right)$ is a triform. Moreover, it follows immediately that, in this case, the group $\mathscr{G}$ of all trimorphisms of $X$ is the symmetric group on $X$. However, the automorphism group $\mathscr{A}$ of $\mathscr{S}[X]$ is isomorphic to the group, under composition, of all homeomorphisms on $X$. To see this, note that the group $\mathscr{U}$ of all units of $\mathscr{S}[X]$ is the group of all homeomorphisms on $X$. Thus, any automorphism $\varphi \in \mathscr{A}$ must map $\mathscr{U}$ isomorphically onto itself. Therefore, according to Theorem 15 [2, p. 248], there exists a homeomorphism $h$ (which must necessarily be unique) of $X$ such that

$$
\varphi(k)=h \circ k \circ h^{-1}
$$

for each $k \in \mathscr{U}$. Just as in the proof of the previous corollary, one shows that the mapping $\Phi$ from $\mathscr{A}$ into $\mathscr{U}$ which is defined by $\Phi(\varphi)=h$ is a monomorphism. Moreover, $\Phi$ is surjective since for any homeomorphism $t$ of $X$, the mapping $\varphi_{t}$ which is defined by

$$
\varphi_{t}(f)=t \circ f \circ t^{-1}
$$

belongs to $\mathscr{A}$ and $\Phi\left(\varphi_{t}\right)=t$. Thus $\mathscr{A}$ is isomorphic to $\mathscr{U}$. However, $\mathscr{U}$ and $\mathscr{G}$ are not isomorphic since the former contains $c$ elements and the latter contains $2^{\circ}$ elements.

\section{Semigroups of closed relations on topological spaces}

Now we apply the results of the previous section to semigroups of closed relations on topological spaces. By a closed relation, we simply mean any binary relation on $X$ which is a closed subset of $X \times X$. It will be convenient to assume throughout the remainder of the paper that all topological spaces discussed are $T_{1}$ spaces.

It so happens that, in general, the composition of two closed relations on a space $X$ need not be a closed relation. This leads us to make the following

Definition (3.1). A space $X$ is a $(5$-space if for each pair $A, B$ of closed relations on $X, A \circ B$ is also a closed relation on $X$. 
The following result characterizes $\mathbb{E}$-spaces within the class of all first-countable spaces.

TheOrem (3.2). A first countable space is a $\mathbb{E}$-space if and only if it is sequentially compact or discrete.

Proof. (Sufficiency). It is evident that a discrete space is a (E-space so suppose $X$ is sequentially compact. Let $A$ and $B$ be two closed relations on $X$ and let $(p, q)$ be a limit point of $A \circ B$. Then since $X$ is first countable, there exists a sequence $\left\{\left(x_{n}, y_{n}\right)\right\}_{n=1}^{\infty} \subset A \circ B$ which converges to $(p, q)$. For each $\left(x_{n}, y_{n}\right)$, there exists a $z_{n} \in X$ such that $\left(x_{n}, z_{n}\right) \in B$ and $\left(z_{n}, y_{n}\right) \in A$. Since $X$ is sequentially compact, there exists a subsequence $\left\{z_{n_{k}}\right\}_{k=1}^{\infty}$ of $\left\{z_{n}\right\}_{n=1}^{\infty}$ which converges to some point $t \in X$. Then $\left\{\left(x_{n_{k}}, z_{n_{k}}\right)\right\}_{k=1}^{\infty}$ converges to $(p, t)$ and $\left\{\left(z_{n_{k}}, y_{n_{k}}\right)\right\}_{k=1}^{\infty}$ converges to $(t, q)$. Since $A$ and $B$ are closed subsets of $X \times X$, we have $(p, t) \in B$ and $(t, q) \in A$. Consequently, $(p, q) \in A \circ B$.

(Necessity). Suppose $X$ is neither sequentially compact nor discrete. We need only produce two closed relations $A$ and $B$ on $X$ such that $A \circ B$ is not closed. Since $X$ is not sequentially compact, there exists a sequence $\left\{x_{n}\right\}_{n=1}^{\infty}$ of distinct points with no convergent subsequence. Since $X$ is first countable and not discrete, there exists a sequence $\left\{y_{n}\right\}_{n=1}^{\infty}$ of distinct points converging to a point $q \in X$ such that $x_{n} \neq y_{m}$ for all $m, n$ and $x_{n} \neq q \neq y_{n}$ for all $n$. Let

Then

$$
A=\left\{\left(x_{n}, y_{n}\right)\right\}_{n=1}^{\infty} \text { and } B=\left\{\left(y_{n}, x_{n}\right)\right\}_{n=1}^{\infty} \text {. }
$$

$$
A \circ B=\left\{\left(y_{n}, y_{n}\right)\right\}_{n=1}^{\infty} .
$$

Neither $A$ nor $B$ have any limit points and are therefore closed. However, $A \circ B$ is not closed since it does not contain the limit point $(q, q)$.

Now let $X$ be a $\mathfrak{E}$-space, let $\mathscr{F}_{X}$ denote the family of all closed subsets of $X$ and let $\mathbb{C}[X]$ denote the family of all closed subsets of $X \times X$. Keeping in mind the blanket assumption that all spaces discussed here are $T_{1}$ spaces, one notes that the triple $\left(X, \mathscr{F}_{X}, \mathbb{E}[X]\right)$ is a triform and, in particular, that $\mathbb{E}[X]$ is a semigroup under composition. If $\left(Y, \mathscr{F}_{X}, \mathfrak{E}[Y]\right)$ is another such triple, the trimorphisms from $X$ onto $Y$ are simply the homeomorphisms from $X$ onto $Y$. Theorem (2.2) now applies and we immediately get

TheOREM (3.3). Let $X$ and $Y$ be $\mathbb{E}$-spaces and let $\mathbb{E}[X]$ and $\mathbb{E}[Y]$ denote the semigroups of all closed relations on $X$ and $Y$ respectively. Then the following statements concerning a bijection $\varphi$ from $\mathbb{E}[X]$ onto $\mathbb{E}[Y]$ are equivalent:

(3.3.1) $\varphi$ is an isomorphism.

(3.3.2) There exists a homeomorphism $h$ from $X$ onto $Y$ such that $\varphi(A)=h \circ A \circ h^{-1}$ for each $A \in \mathbb{E}[X]$. 
(3.3.3) There exists a homeomorphism $h$ from $X$ onto $Y$ such that $\varphi(A)=\{(h(x), h(y)):(x, y) \in A\}$ for each $A \in \mathbb{E}[X]$.

We define an endomorphism $\varphi$ of an arbitrary semigroup $\mathscr{S}$ to be inner if there exist elements $a, b \in \mathscr{S}$ such that $\varphi(x)=a x b$ for each $x \in \mathscr{S}$. M. L. Vitanza, has shown in [5, p. 1079], Theorem 1 that if the inner endomorphism $\varphi$ is, in fact, an inner automorphism, then the semigroup $\mathscr{S}$ contains an identity and the elements $a$ and $b$ are inverses of each other relative to that identity.

Now suppose we take the spaces $X$ and $Y$ to be identical in Theorem (3.3). If, in addition, $X$ is Hausdorff, then the homeomorphisms $h$ and $h^{-1}$ of (3.3.2) are both elements of $\mathbb{C}[X]$ and we have the following

Corollary (3.4). Let $X$ be a Hausdorff $\mathbb{C}$-space. Then every automorphism of the semigroup $\mathbb{C}[X]$ is inner.

Since every discrete space is a $\mathbb{E}$-space, the latter corollary generalizes the theorem in [4] which states that every automorphism of the semigroup of all binary relations on a nonempty set is inner. Actually, composition is defined in [4] in such a manner that the semigroup discussed there is antiisomorphic to the corresponding one discussed here. Of course, if any semigroup has the property that all automorphisms are inner, the same is true for any anti-isomorphic copy.

We have the following result on the automorphism group of $\mathbb{E}[X]$.

Corollary (3.5). Let $X$ be a $\mathbb{E}$-space. Then the automorphism group of the semigroup $\mathbb{C}[X]$ is isomorphic to the group, under composition, of all homeomorphisms mapping $X$ onto $X$.

Proof. According to Theorem (3.3), for each automorphism $\varphi$ of $₫[X]$ there exists a (necessarily unique) homeomorphism $h$ of $X$ such that $\varphi(A)=h \circ A \circ h^{-1}$ for each $A \in \mathbb{E}[X]$. We define a mapping $\Phi$ from the automorphism group $\mathscr{A}$ of $\mathbb{C}[X]$ into the group $\mathscr{G}$ of all homeomorphisms on $X$ by $\Phi(\varphi)=h$. One shows just as in the proof of Corollary (2.14) that $\Phi$ is a monomorphism. To see that $\varphi$ is surjective in this case, note that for any homeomorphism $h$, the mapping $\psi$ defined by $\psi(A)=h \circ A \circ h^{-1}$ for each $A \in \mathbb{E}[X]$ is an automorphism of $\mathbb{E}[X]$ and $\Phi(\psi)=h$.

Just as Corollary (3.4) generalizes the theorem in [4], this latter result generalizes the corollary in [4] which states that the automorphism group of the semigroup of all binary relations on a set is isomorphic to the symmetric group on $X$.

We remark in closing that in a later paper, we intend to investigate topological properties which are such that if $A, B \subset X \times X$ have the given property, then $A \circ B$ also has the property. This will allow us to apply Theorem (2.2) and related results to semigroups of binary relations on a topological space, all of which have a given topological property. 


\section{References}

[1] A. H. Clifford and G. B. Preston, The algebraic theory of semigroups, Mathematical Surveys, Number 7 (Amer. Math. Soc., 1961).

[2] N. J. Fine and G. E. Schweigert, 'On the group of homeomorphisms of an arc', Annals of Math. 62 (1955) 237-253.

[3] E. S. Ljapin, Semigroups, Vol. 3, Translations of Mathematical Monographs (Amer. Math. Soc., 1963).

[4] K. D. Magill, Jr., 'Automorphisms of the semigroup of all relations on a set', Canad. Math. Bull., Vol. 9, No. 1, (1966), 73-77.

[5] M. L. Vitanza, 'Mappings of semigroups associated with ordered pairs', Amer. Math. Monthly 73 (1966), 1078-1082.

State University of New York at Buffalo

Buffalo, New York 